\title{
Improving Maternity Care with Business Intelligence
}

\author{
Patrícia Loreto, Francisca Fonseca \\ and Ana Morais \\ University of Minho \\ \{a71934, a71702,a70484\}@alunos.uminho.pt
}

\author{
Hugo Peixoto, António Abelha \\ and José Machado \\ Algoritmi Research Center \\ \{hpeixoto, abelha, jmac\}@di.uminho.pt
}

\begin{abstract}
The aim of this paper is to develop clinical indicators for obstetrics through the use of Business Intelligence (BI) tools, since valid and reliable clinical indicators can help measuring quality of healthcare services and support decision-making processes. This paper gives an overview of concepts related to Health Information Systems (HIS) and BI, along with some related work to highlight the advantages that BI solutions can bring when applied to healthcare. In this paper is also presented the data warehousing and the ETL process, that was necessary for the development of indicators and which is usually hidden from endusers, is described. The indicators were developed using Power $B I$ and were analysed and compared with reference values from both national and international health reports. The discussion of the developed indicators made it possible to measure the quality of the obstetrics service, to identify the problematic areas and to decide whether improvement measures should be taken.

Keywords-Health Information Systems (HIS), Interoperability, Business Intelligence (BI), Data Warehousing, ExtractTransform-Load (ETL), Power BI, Indicators, Obstetrics.
\end{abstract}

\section{INTRODUCTION}

\section{A. Health Information Systems}

The Information Technology (IT) today plays an important role in healthcare institutions, due to the complex nature of this kind of organizations and the large amount of data that is typically produced [1], [2].

Health Information Systems (HIS) are Information Systems that store, process and exchange data, information and knowledge in a healthcare institution which are required for planning, organizing, executing and evaluating the healthcare services provided. Therefore, the main goal of HIS is to improve the efficiency and quality of healthcare which leads to several advantages such as cost reductions, optimization of resources and reduction of the number and incidence of medical errors [1]. More specifically, HIS can manage information about patients and enable the extraction of clinical and management indicators. Subsequently, these indicators can help professionals making more informed decisions, therefore improving the planning and provision of healthcare services [1].

In Portugal, among the great variety of HIS implemented, the following can be highlighted [3], [4]:

- $\mathrm{SONHO}$ - this information system is responsible for the administrative management of patients, commonly used in hospitals;
- SINUS - like SONHO, this information system is responsible for the administrative management of patients, but is commonly used in primary care units;

- SCLINICO - this information system is oriented to the activity of health professionals, having a version for doctors and another for nurses;

- $R N U$ - this is a centralized information system that contains the list of all Portuguese patients and allows a unique and unambiguous identification of patients;

- $C T H$ - the aim of this information system is to manage the waiting lists for medical appointments;

- SIGIC - similar to CTH, but manages the waiting lists for surgeries.

However, even though HIS contribute to increase the quality of healthcare services, the information sources are heterogeneous, distributed, large and complex, which means that the different HIS must communicate to exchange information and make it available at any place and at any time [5]. Consequently, HIS must fulfil certain requirements such as interoperability, security, scalability and reliability in data storage and processing [1].

\section{B. Interoperability}

Interoperability can be defined as the capacity that heterogeneous and independent HIS and computer applications have to communicate and exchange data in an accurate, effective and consistent manner and to use the information that has been exchanged, in order to achieve a mutual benefit and without any special effort from the users [1].

The main goal of interoperability in healthcare is to connect the different applications and data so that the information can be shared by the entire healthcare environment and accessed by health professionals and patients whenever and wherever they need it [5].

Nevertheless, to ensure interoperability in the healthcare environment remains a challenge mainly due to the great number of different HIS that healthcare organizations have. Even within the same organization, it is common to have specific HIS for each medical service or speciality and these systems are developed based on various technological platforms and different programming languages. This reality is one of the main reasons why interoperability of information systems in healthcare institutions is still very poor and difficult to 
implement [1].

There are several methodologies that already exist to promote interoperability among the HIS and that are based on common communication architectures and mainstream standards [5]. Indeed, standards in healthcare are considered one of the main ways to ensure interoperability and these include standards for messages (HL7), terminologies (SNOMED-CT), clinical information and patient records (OpenEHR) and images (DICOM) [1].

To ensure interoperability among the HIS, there is also the AIDA platform, which stands for Agency for Integration Diffusion and Archive of Medical Information and is implemented in several hospitals in Portugal. AIDA is a central platform that facilitates the dissemination and integration of information generated in hospital environments by performing tasks such as communicating with the heterogeneous systems, sending and receiving information (e.g. medical or clinical reports, images, collections of data, prescriptions), managing and storing the information and answering to information requests [5], [6], [2], [7].

\section{Electronic Health Record}

As a component of the HIS, the Electronic Health Record (EHR) consists of the chronological agglomeration of a patient's clinical information from the received care, catalogued by the place and professionals involved in the provided care. The EHR contains this information in a computer processable form, that can be stored and transmitted securely, and accessed by multiple authorized users [1], [8]. Thus, the EHR has three main components - repository, secure storage and exchange of clinical information, and authorized accessibility - which are fundamental for implementing the EHR in the clinical practice [8].

Since healthcare organizations operate in a dynamic environment, the EHR is developed with interoperable standards so that the capture, storage and retrieval of both structured and unstructured clinical data sets can be standardized [8].

The main advantages of using the EHR include the quick access to useful information of patients needed for decisionmaking at the point of care, the ability to allow simultaneous consultation and edition of a patient's information by clinicians, the promotion of data security and confidentiality, the integration of different information resources (medical images, laboratory tests, and others), centralization and constantly updated information and promotion of information structuring according to medical standards [1]. The EHR is undoubtedly a valuable element in supporting healthcare services, improving patient safety and promoting an evidence-based medical practice [8].

\section{Business Intelligence}

Data mining algorithms can be applied to extract relevant data from the EHR and the use of Business Intelligence (BI) has the potential to operationalize the EHR repository in supporting evidence-based practice and improving the quality and safety of healthcare services [8], [6], [9].
BI refers to a group of data analysis tools that includes a broad category of methodologies, applications and technologies for collecting, storing, manipulating, analysing and providing access to data in order to help organizations to make better and faster decisions. BI includes many software tools for data warehousing, Extract-Transform-Load (ETL), database query and reporting, multidimensional/online analytical processing (OLAP) data analysis and data mining [8], [6].

BI tools have the ability to transform information into knowledge and can be used as middleware between transactional applications and decision support systems, shortening the time between data acquisition and decision making. Other benefits of BI include the increased autonomy and flexibility users can have with BI when creating reports and analysis due to its ease of use, the improved decision support and operational efficiency. These benefits are of great interest to healthcare IT professionals [8], [6].

Applying BI to the EHR enable healthcare providers to extract quality and meaningful information from it, therefore facilitating real-time decision making, evidence-based practice and improving patient outcomes. Moreover, it allows healthcare professionals to obtain the required clinical information in an efficient and easy way [8], [10], [6], [11].

However, integrating BI with the EHR requires, at least, the availability of a clinical data warehouse, data sources with good quality and query and reporting tools [8].

A data warehouse is a system that extracts, cleans, conforms, and delivers source data into a dimensional data store, supporting and implementing querying and analysis useful for decision-making [12]. Data warehouse systems are built to support users analysing a large amount of data that comes from many and heterogeneous data sources which are commonly operational transaction systems that register the daily activities of organizations [13].

The purpose of a data warehouse is to collect data from different sources and to provide its end-users access to this information. However, the different information structures that result from collecting data from various sources or even the lack of quality of some data composes a set of problems which need to be overcome [14]. This is possible due to the processes of ETL whose mission is to get data out of the source, clean and transform this data and then load it into the data warehouse [12], [15].

OLAP technologies can contribute to the successful implementation of a data warehouse since OLAP interfaces provide a simple, yet flexible navigation and presentation environment that represents the structure of real world data stored in the form of multidimensional tables or data cubes. A data cube can be implemented as a relational star schema and has two types of components: facts, which are tables populated with numeric attributes, and dimensions, that consist of many categorical or textual attributes [13].

Dimensions can have associated hierarchies which specify different aggregation levels and allow users to "slice and dice" a data cube to form customized data sets for further analysis. In fact, OLAP interfaces take advantage of the predictable 
structure of data cubes (composed by dimensions and facts) to provide a graphical query environment where users can also navigate through a cube depending on the wanted level of granularity [13].

\section{E. Business Intelligence Visualization Tools}

Traditional data visualization tools are not appropriate for BI applications since data sets are large and constantly updating. Besides, one of the main requisites for data visualization in BI is that the visualization must be interactive and without noticed delays, which is usually not accomplished in traditional tools [16], [6].

To help overcoming the previously presented problems, several tools have been developed. Some BI visualization tools are listed in Table I and are compared considering relevant criteria. The used criteria were [16], [17], [6]:

- Open-source tool - if the tool is open source or not;

- Interactive visualization - if the created visualizations are interactive or not;

- Available versions - if it has a desktop, online or mobile version;

- Available tutorials - if there are any online tutorials available;

- Linux - if it works on Linux or not;

- Windows - if it works on Windows or not;

- Unix - if it works on Unix or not;

- Report - if reporting is allowed;

- Dashboard - if it allows the creation of dashboards or not;

- Graphics - if it has a set of available graphs or not.

\section{F. Indicators}

Indicators are variables that assess the quality of an organization [24]. Quality assessment has become increasingly important over the years to all the stakeholders in this field and indicators are a way of measuring it [25]. In healthcare organizations, they can either be classified as performance or clinical indicators [25], [26].

Performance indicators offer information about the quality of an institution or system and help guiding future decisions [24]. A clinical indicator is a variable that measures clinical activities or medicine-related activities and describes the performance of a specific health care process and respective results [25], [27], [26]. This type of indicators make it possible to [25], [26]:

- Compare different healthcare institutions;

- Report the quality of care;

- Define priorities;

- Assist in accountability, regulation and accreditation;

- Bolster quality improvement;

- Justify patients decisions regarding the choice of providers;

- Offer transparency in health care.

This paper will focus on this type of indicators.

In obstetrics, these indicators are extremely important, because this medical specialty involves multiple procedures, there are two lives involved and it is one of the most affected areas by patient complaints [28].

\section{BACKGROUND AND RELATED WORK}

\section{A. Related Work}

Given the constant progression that the healthcare sector nowadays has, the applicability of BI solutions in this sector is increasing, since they can solve problems such as the lack of access and ability to use data collected from non-integrated traditional information systems, and support decision-making processes. Thus, in this section some existing works of applying BI tools for supporting decision-making in healthcare are presented.

Brandão et al. (2014) developed a global BI platform that can be used in Maternity Care Hospitals for decision-making support. The case of study was Centro Materno Infantil do Norte (CMIN) and it involved the construction of two data warehouses - one for Gynaecology and another for Obstetrics, requiring as well a ETL process to extract, transform and load data from databases used in CMIN into the data warehouses [11].

The main goal of this BI platform was to facilitate the work of health professionals who are responsible for obtaining reports, allowing them to obtain indicators related to the information registered during medical procedures. Therefore, this platform demonstrated to increase the autonomy and flexibility of health professionals when it comes to creating reports, also enabling a faster and easier analysis of clinical data and supporting clinical decision-making. It is also important to mention that this project can be useful for implementing BI platforms in other maternity care institutions [11].

Prevedello et al. (2010) tested the usability of open-source BI tools for Radiology, which is a health service commonly known to have its information available in multiple systems [29].

Aiming to create new key performance indicators specific to Radiology, they developed a data warehouse prototype using open-source tools, namely the ETL component of Pentaho was used for creating the Radiology Data Warehouse and a MySQL Server database was used to connect to Pentaho. Henceforth, this study demonstrated that converting large relational databases into a multidimensional format can indeed result in performance improvements when reports need to be generated. It also demonstrated that open-source tools can be used to create a micro-database from various departmental information sources, which can be useful in the selection of new key performance indicators to monitor activities and processes within the same department [29].

Ali et al. (2016) developed a BI solution to empower staff and management for strategic decision making through data warehousing based on knowledge management tools. The case of study in this work was the rehabilitation of hip fracture care, where the use of the resulting BI solution leads to quality of rehabilitation care improvements, optimization of patient's 
Table I

COMPARISON OF BUSINESS INTELLIGENCE VISUALIZATION TOOLS [16], [17], [18], [19], [20], [21], [22], [23]

\begin{tabular}{lllllll}
\hline & Tableau & Power BI & Plotly & Gephi & Excel 2016 & SpagoBI \\
\hline Open-source & No & No & Yes & Yes & No & Yes \\
Linux & No & No & Yes & Yes & No & Yes \\
Windows & Yes & Yes & Yes & Yes & Yes & Yes \\
Unix & Yes & No & Yes & Yes & Yes & Yes \\
Desktop version & Yes & Yes & No & Yes & Yes & Yes \\
Online version & Yes & Yes & Yes & No & Yes & Yes \\
Mobile Application & Yes & Yes & No & No & Yes & No \\
Tutorials available & Yes & Yes & Yes & Yes & Yes & Yes \\
Interactive visualization & Yes & Yes & Yes & Yes & Yes & Yes \\
Reports & Yes & Yes & Yes & No & Yes & Yes \\
Dashboards & Yes & Yes & Yes & No & Yes & Yes \\
Graphics & Yes & Yes & Yes & No & Yes & Yes \\
\hline
\end{tabular}

workflow, reduction of waiting times for surgery and reduction of length of hospital stay for patients. These successes were due to the ability to visualize data in timely ways through the data warehouse and OLAP cubes [30].

By providing up to date and accurate data through the utilization of BI applications (e.g data warehouse modelling, integration, analysis and reporting services) to healthcare databases, this solution allowed health professionals to focus their efforts on the key system areas with the greatest opportunities for improvement [30].

Undoubtedly, decision support tools such as data marts, OLAP cubes and data mining techniques can provide a solid foundation for clinical data warehouses since presenting data in a multi-dimensional format allows the analysis of large amounts of data in short periods of time [30].

\section{B. Obstetrics}

Obstetrics is considered a sub-speciality of surgery that provides surgical care for women during pregnancy and childbirth. Obstetricians also handle postnatal care and can deal with a wide range of situations related to the reproductive system [31].

Obstetricians keep in contact with future mothers throughout their pregnancy during appointments to detect potential problems and to advise patients on which steps can be taken to prevent any pregnancy issues. Then, when a woman experiences signs of labour, her obstetrician and other support staff perform the required delivery procedures. Once the infant is born, the doctor assesses the health conditions of both mother and child to make sure that no emergent conditions require treatment [32]. Namely, in order to carefully check each newborn baby for signs or complications, the health professional checks for the baby's vital signs (temperature, pulse, breathing rate), performs an APGAR scoring test at 1,5 and 10 minutes after birth, which can help finding breathing problems and other health issues, and then other measures such as birth weight, head circumference and length are also taken [33].

Thus, the main purpose of obstetrics is to assure the wellbeing of the pregnant mother and the baby and to promote the success of the delivery process. The delivery procedures can be mainly classified as vaginal deliveries or caesarean sections, considering that a caesarean section is a surgical procedure that is performed in risk pregnancies and that vacuum extraction and obstetric forceps are used when vaginal deliveries require additional support [34].

Obstetrics can be extremely stressful and doctors tend to work long and irregular hours since labour is not easily predictable and bad outcomes in the delivery room can be emotionally draining [32]. For these reasons, having a BI system that produces indicators, while making data easier to be visualized and accessed, can be of great interest for clinicians since it enables them to manage their time and resources better and to reduce medical errors.

The data we have considered in this paper is mainly focused on the moment of the delivery. Therefore, it is considered information about the mother and about the newborn at the moment of childbirth and also information about the delivery process.

\section{Methodology}

In this section, we present a description of the ETL process, the construction of the multidimensional model and the development of the indicators.

\section{A. Extract}

The first step was to obtain the appropriate data from individual data sources like servers [14], [35], [10]. The data used on this paper was extracted from the obstetrics service of a Portuguese hospital.

Among all the data collected on childbirth, there was information about the mother and about the newborn. Each newborn identification number was associated not only with the newborn sequential and episode number but also with his mother episode number. Besides, physical information about the newborn and if he survived to childbirth or needed resuscitation were associated to the newborn identifying number too. The characterization of the childbirth which resided on its date and time and a brief description of it was together with the information mentioned before. Moreover, a more detailed description of the childbirth, with information about anaesthesia and degree of laceration, was presented in $\mathrm{xml}$ documents associated to the mother process, episode and 
sequential numbers along with her date of birth. Between all the data extracted, the informations mentioned above were the ones that drew attention.

\section{B. Transform}

Once the target of the ETL processes is the dimensional data structures, this next phase started with the construction of the multidimensional model. The use of dimensional data models entails some advantages such as the simplicity of their creation, stability in the presence of changing data environments, and ease of understanding by end-users. They are the foundation for constructing all forms of OLAP cubes [12]. Besides, the construction of the multidimensional model allowed to indentify which of the previously extracted columns were of interest and which should be discarded. The muldimensional model was constructed in a relational star schema and its fact table is shown in Figure 1.

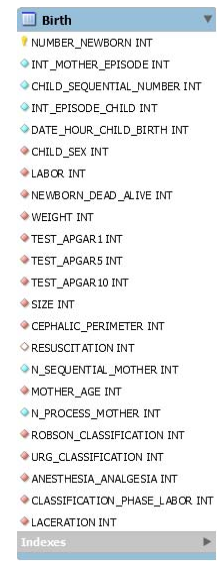

Figure 1. Fact table of the multidimensional model

Since dimensional models are built around measurement processes, the fact table corresponds to the birth event and includes foreign keys referring to the dimensions attached to the fact table. All the dimensional attributes are presented in Table II and are textual [12].

As presented by Vassiliadis (2009), one of the inherent problems is the lack of quality that the data coming from the sources suffer. This lack of quality comes from misspellings, missing information (for example, some of the babies did not have any information on the mother or vice versa), and inconsistent values too. In order to provide end-users data as clean, complete, and truthful as possible, this kind of "noise" had to be removed from the data [10].

Additionally, some decisions on the representation of data were made. The mothers' age was distributed by three intervals based on the literature found: adolescent mother $(\leq 19)$, aged mother $(\geq 35)$ and normal age mother. Weight, height and head perimeter were represented in terms of percentiles defined by the Directorate-General for Health (DGS) and the results of the APGAR tests were also presented over the intervals $[0,3],[4,6]$, and $[7,10]$.
Table II

DIMENSIONS OF THE MULTIDIMENSIONAL MODEL

\begin{tabular}{cc}
\hline Dimension & Attributes \\
\hline Dim_weight & idDim_weight; Percentile \\
\hline Dim_size & idDim_size; Percentile \\
\hline Dim_mother_age & idDim_mother_age; Percentile \\
\hline Dim_test_APGAR1 & idDim_test_APGAR1; Interval \\
\hline Dim_test_APGAR5 & idDim_test_APGAR5; Interval \\
\hline Dim_test_APGAR10 & idDim_test_APGAR10; Interval \\
\hline Dim_CLURG & Group; Description \\
\hline Dim_phase_labor & Group; Description \\
\hline Dim_resuscitation & Degree; Description \\
\hline Dim_cephalic_perimeter & idDim_cephalic_perimeter; Percentile \\
\hline Dim_newborn_dead_alive & idDim_newborn; Description \\
\hline Dim_anaesthesia_analgesia & idDim_anaesthesia; Description \\
\hline Dim_labor & idDim_labor; Description \\
\hline Dim_Robson_classification & Group; Description \\
\hline Dim_sex & idDim_sex; Description \\
\hline
\end{tabular}

Some of the data of interest, for example the degree of laceration or information on anaesthesia, were in $\mathrm{xml}$ documents. To draw this information out in this ETL phase would not be feasible due to the amount of data that would need to be taken manually.

\section{Load}

Through MySQL Workbench it was possible to create the tables by loading the multidimensional model. Although the treated data were loaded to these tables, the xml documents were loaded to external tables to the multidimensional model. These last tables and the fact table have in common the newborn number (primary key). This approach was chosen to ease the extraction process of data from xml documents. This way, it is only needed to update the fact table with the data extracted from the second column (xml data) of these external tables.

\section{Development of Indicators}

With the constructed data set it is now possible to use one of the previously mentioned Business Intelligence visualization tools to develop indicators from the data.

We decided to use Power BI mainly because:

- It is a recent tool with a large community;

- Its dashboards can be connected to a real-time data source which enables automatic updates;

- It allows access to all data from a single location.

To determine which indicators we would develop, we decided to look for both Portuguese and international health reports. This has the advantage of allowing a comparison with real statistics and goals. 


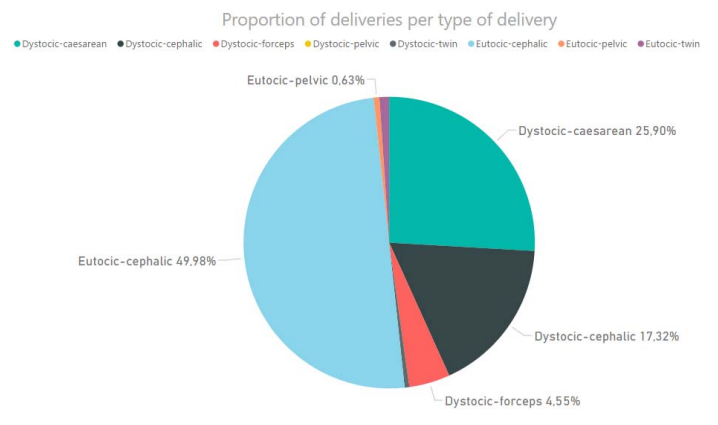

Figure 2. Proportion of deliveries per type of delivery

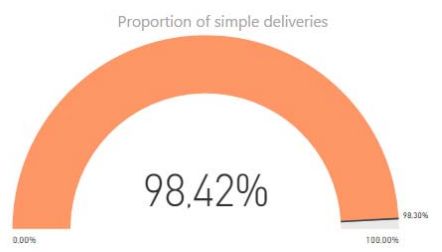

Figure 3. Proportion of simple deliveries

The developed indicators, the respective document where they are mentioned and the reference value are presented in Table III.

We decided to use the gauge chart in most of the indicators, as it allows a quick comparison between the obtained value and the reference value. For the indicator Proportion of deliveries per degree of laceration, we opted to use the pie chart, because in this case there are multiple proportions that need comparison.

\section{RESUlts AND Discussion}

In this section, the developed indicators are presented and their resulsts are discussed. This analysis allows the identification of the areas that need special care and additional measures.

\section{A. General Overview}

The data set is composed by a total of 2529 deliveries, of which 2518 were live births. In Figure 2 is a pie chart with a general overview of the deliveries and their respective type.

\section{B. Proportion of simple deliveries}

Simple deliveries are deliveries in which only one birth occurs [36].

As it can be seen in Figure 3, the proportion of simple deliveries in this data set $(98.42 \%)$ is similar to the reference value $(98.30 \%)$. This indicates that this sample is in line to what happens nationally.

\section{Proportion of eutocic deliveries}

When the delivery is not done with instrumental intervention and with or without episiotomy, the delivery is considered an eutocic delivery [36].

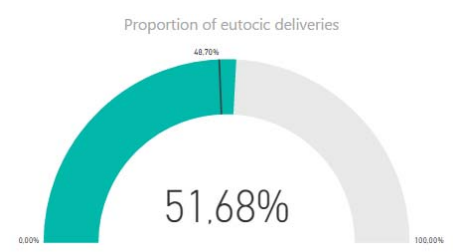

Figure 4. Proportion of eutocic deliveries

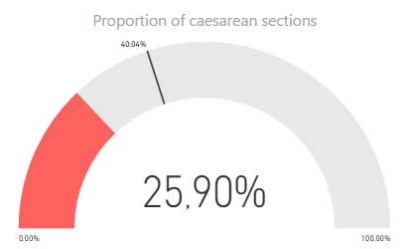

Figure 5. Proportion of caesarean sections

As seen in Figure 4, The Proportion of eutocic deliveries obtained $(51.68 \%)$ is similar to the reference value $(48.70 \%)$ so this does not indicate that something needs to be changed.

\section{Proportion of caesarean sections}

The caesarean section is a procedure where a fetus is extracted by incisions in the abdominal wall and in the uterine wall [36].

The goal for 2016 in Portugal was 40,04\% and through the examination of the Figure 5 it is possible to conclude that the Proportion of caesarean sections is visibly bellow the goal and, therefore, there is no need to implement any reduction measures.

\section{E. Proportion of live births in adolescent women}

The indicator Proportion of live births in adolescent women measures the rate of live births in which the mother's age is below 20 years [36].

In Figure 6, it is possible to see that the obtained value $(2.86 \%)$ is similar to the reference value $(2.60 \%)$, which is slightly higher than the 2016 goal for Portugal. This indicates that probably there is no need to develop further prevention measures, but maybe it is necessary to intensify the already ongoing measures and to pay attention to this indicator evolution.

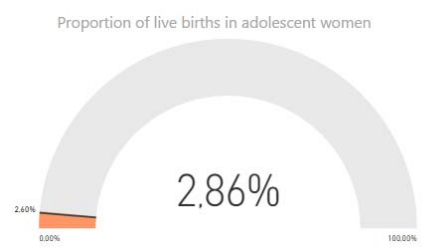

Figure 6. Proportion of live births in adolescent women 
Table III

DEVELOPED INDICATORS, RESPECTIVE DOCUMENT AND REFERENCE VALUE

\begin{tabular}{|c|c|c|}
\hline Indicator & Document & Reference value \\
\hline Proportion of simple deliveries & $\begin{array}{l}\text { Estatísticas da Saúde } 2015 \\
\text { (Statistics Portugal, 2017) }\end{array}$ & $98,30 \%$ \\
\hline Proportion of eutocic deliveries & $\begin{array}{l}\text { Estatísticas da Saúde } 2015 \\
\text { (Statistics Portugal, 2017) }\end{array}$ & $48,70 \%$ \\
\hline Proportion of caesarean sections & $\begin{array}{c}\text { Plano Nacional de Saúde 2012-2016: Indicadores e Metas em Saúde } \\
\text { (Directorate-General of Health, 2012) }\end{array}$ & $40,04 \%$ (goal for 2016) \\
\hline Proportion of live births in adolescent women & $\begin{array}{c}\text { Plano Nacional de Saúde 2012-2016: Indicadores e Metas em Saúde } \\
\text { (Directorate-General of Health, 2012) }\end{array}$ & $2,60 \%$ (goal for 2016) \\
\hline $\begin{array}{l}\text { Proportion of deliveries with anaesthetic or } \\
\text { analgesic use before or during delivery }\end{array}$ & $\begin{array}{c}\text { Hospital Maternity Activity } \\
\text { (National Health System, 2016) }\end{array}$ & $59,40 \%$ \\
\hline $\begin{array}{c}\text { Proportion of deliveries per degree of } \\
\text { laceration }\end{array}$ & $\begin{array}{l}\text { Australia's mothers and babies } 2014 \\
\text { (Australian Institute of Health and Welfare, 2016) }\end{array}$ & $\begin{array}{c}\text { Degree } 1-23,00 \% \\
\text { Degree } 2-28,00 \% \\
\text { Degree } 3 \text { or } 4-3,00 \%\end{array}$ \\
\hline $\begin{array}{c}\text { Proportion of live births that required } \\
\text { resuscitation }\end{array}$ & $\begin{array}{l}\text { Australia's mothers and babies } 2014 \\
\text { (Australian Institute of Health and Welfare, 2016) }\end{array}$ & $20,00 \%$ \\
\hline
\end{tabular}

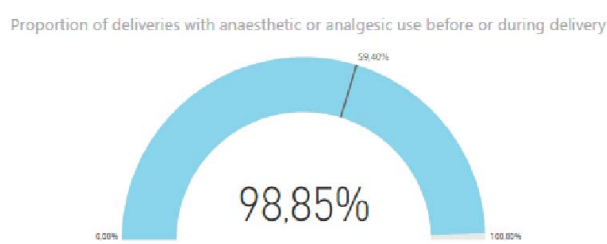

Figure 7. Proportion of deliveries with anaesthetic or analgesic use before or during delivery

F. Proportion of deliveries with anaesthetic or analgesic use before or during delivery

In this case, it is clear by the analysis of the Figure 7 that further measures are needed to reduce the Proportion of deliveries with anaesthetic or analgesic use before or during delivery. These measures may include the adoption of guidelines on epidurals that state that all women should be advised of the advantages and risks of epidurals [37].

\section{G. Proportion of deliveries per degree of laceration}

Lacerations are injuries that occur during delivery and that affect tissues from the female reproductive system. These lesions vary in degree of severity, with degree 1 lesions being the least severe and degree 4 lesions being the most severe [38].

Comparing the reference values with the obtained values (Figure 8), it is possible to observe that in our population the proportion of lacerations is smaller, which can be a sign of less complications during delivery.

\section{H. Proportion of live births that required resuscitation}

According to the report Australia's mothers and babies 2014 (Australian Institute of Health and Welfare, 2016), "Resuscitation is undertaken to establish independent respiration and

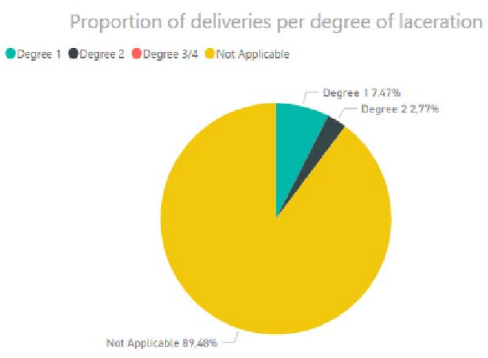

Figure 8. Proportion of deliveries per degree of laceration

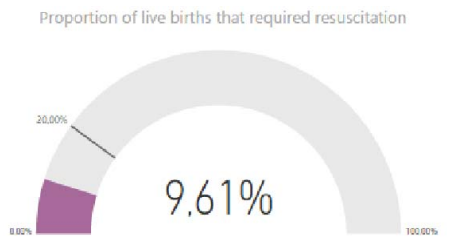

Figure 9. Proportion of live births that required resuscitation

heartbeat or to treat depressed respiratory effect and to correct metabolic disturbances".

In the studied sample, $9,61 \%$ of live births required at least one type of resuscitation. This value is less than the reference value (20\%), which may point that, for example, less complications happened during delivery.

\section{CONCLUSIONS AND Future WORK}

This paper aimed to demonstrate the importance of BI tools, mainly indicators, on health information systems. As it has already been said, through the use of indicators it is possible to assess the quality of a certain service which, in this study, is Obstetrics. They not only measure changes, but indicators 
also serve as a guide for future actions to promote quality. The use of these indicators in a database at a national level would allow hospitals to grow as presented by Sadeghi-Bazargani et al. (2014).

Similarly, using clinical indicators in this study made it possible to evaluate the quality of the obstetrics service at the hospital concerned. By comparing the indicators obtained with a national or international reference value, identification of the areas that required improvement was possible. Therefore, the hospital, now knowing exactly where to act, is able to establish a successful plan of action and focus on quality improvement of patient care.

Although indicators bring all these advantages to health organizations, there is a laborious work before their construction that is hidden from end-users. All the data has to go through a BI tool, an ETL system, the information extracted has to be treated before loaded to guarantee the quality of the data and also their reliability. However, the extraction referred on this article was "handmade" which constitutes an obstacle to keep data, and consequently indicators, updated. The next step would be to modify the first phase of the ETL process by making the extraction process automatic.

\section{ACKNOWLEDGMENTS}

This work has been supported by Compete: POCI-010145-FEDER-007043 and FCT within the Project Scope UID/CEC/00319/2013.

\section{REFERENCES}

[1] J. Machado and A. Abelha, Applying Business Intelligence to Clinical and Healthcare Organizations. IGI Global, 2016.

[2] A. Pereira, F. Marins, B. Rodrigues, F. Portela, M. F. Santos, J. Machado, F. Rua, Á. Silva, and A. Abelha, "Improving quality of medical service with mobile health software," Procedia Computer Science, vol. 63, pp. 292-299, 2015.

[3] V. M. O. Pinheiro, "Arquitetura empresarial do centro hospitalar do alto ave," Ph.D. dissertation, 2015.

[4] E. SPMS - Serviços Partilhados do Ministério da Saúde, http://spms. min-saude.pt/product/, [Online; accessed 24-April-2017].

[5] L. Cardoso, F. Marins, C. Quintas, F. Portela, M. Santos, A. Abelha, and J. Machado, "Interoperability in healthcare," Cloud Computing Applications for Quality Health Care Delivery, pp. 78-101, 2014.

[6] A. Brandão, E. Pereira, M. Esteves, F. Portela, M. F. Santos, A. Abelha, and J. Machado, "A benchmarking analysis of open-source business intelligence tools in healthcare environments," Information, vol. 7, no. 4, p. 57, 2016.

[7] A. Novo, J. Duarte, F. Portela, A. Abelha, M. F. Santos, and J. Machado, "Information systems assessment in pathologic anatomy service," in New Contributions in Information Systems and Technologies. Springer, 2015, pp. 199-209.

[8] W. Bonney, "Applicability of business intelligence in electronic health record," Procedia-Social and Behavioral Sciences, vol. 73, pp. 257-262, 2013.

[9] D. Coelho, J. Miranda, F. Portela, J. Machado, M. F. Santos, and A. Abelha, "Towards of a business intelligence platform to portuguese misericórdias," Procedia Computer Science, vol. 100, pp. 762-767, 2016.

[10] M. F. S. J. M. A. A. Ana Pereira, Filipe Portela and F. Rua, "Pervasive business intelligence in intensive medicine," in eTELEMED 2017: The Ninth International Conference on eHealth, Telemedicine, and Social Medicine, 2017, pp. 37-41.

[11] A. Brandão, E. Pereira, F. Portela, M. Santos, A. Abelha, and J. Machado, "Real-time business intelligence platform to maternity care," in Biomedical Engineering and Sciences (IECBES), 2014 IEEE Conference on. IEEE, 2014, pp. 379-384.
[12] R. Kimball and J. Caserta, The Data Warehouse? ETL Toolkit: Practical Techniques for Extracting, Cleaning, Conforming, and Delivering Data. John Wiley \& Sons, 2011.

[13] M. C. Tremblay, R. Fuller, D. Berndt, and J. Studnicki, "Doing more with more information: Changing healthcare planning with olap tools," Decision Support Systems, vol. 43, no. 4, pp. 1305-1320, 2007.

[14] P. Vassiliadis, "A survey of extract-transform-load technology," International Journal of Data Warehousing and Mining (IJDWM), vol. 5, no. 3, pp. 1-27, 2009.

[15] J. Machado, E. Silva, and A. Abelha, "Business intelligence platform for nosocomial infection incidence," Indian Journal of Science and Technology, vol. 10, no. 10, 2017.

[16] S. M. Ali, N. Gupta, G. K. Nayak, and R. K. Lenka, "Big data visualization: Tools and challenges."

[17] M. Tereso and J. Bernardino, "Open source business intelligence tools for smes," in Information Systems and Technologies (CISTI), 2011 6th Iberian Conference on. IEEE, 2011, pp. 1-4.

[18] T. Software, https://www.tableau.com/, [Online; accessed 23-April2017].

[19] P. BI, https://powerbi.microsoft.com/pt-br/, [Online; accessed 23-April2017].

[20] Plotly, https://plot.ly/, [Online; accessed 23-April-2017].

[21] Gephi, https://gephi.org/, [Online; accessed 23-April-2017].

[22] Excel, https://products.office.com/pt-pt/excel, [Online; accessed 23April-2017].

[23] SpagoBI, https://www.spagobi.org/, [Online; accessed 23-April-2017].

[24] R. Askari, M. Shafii, and N. Baghian, "Comparing performance indicators of obstetrics and gynecology ward at yazd educational hospitals with expected limits of indicators, 2015," Osong Public Health and Research Perspectives, vol. 7, no. 3, pp. 197-204, 2016.

[25] J. Mainz, "Defining and classifying clinical indicators for quality improvement," International Journal for Quality in Health Care, vol. 15, no. 6, pp. 523-530, 2003.

[26] _ - "Developing evidence-based clinical indicators: a state of the art methods primer," International Journal for Quality in Health Care, vol. 15, no. suppl 1, pp. i5-i11, 2003.

[27] H. Sadeghi-Bazargani, M. Farhoudi, S. Hajebrahimi, M. NaghaviBehzad, Z. Sohrabnavi, and S. Azami-Aghdash, "A systematic review on clinical indicators, their types and codification processes," Journal of Clinical Research \& Governance, vol. 4, no. 1, 2014.

[28] R. Boulkedid, C. Alberti, and O. Sibony, "Quality indicator development and implementation in maternity units," Best Practice \& Research Clinical Obstetrics \& Gynaecology, vol. 27, no. 4, pp. 609-619, 2013.

[29] L. M. Prevedello, K. P. Andriole, R. Hanson, P. Kelly, and R. Khorasani, "Business intelligence tools for radiology: creating a prototype model using open-source tools," Journal of digital imaging, vol. 23, no. 2, pp. 133-141, 2010.

[30] O. Ali, P. Crvenkovski, and H. Johnson, "Using a business intelligence data analytics solution in healthcare," in Information Technology, Electronics and Mobile Communication Conference (IEMCON), 2016 IEEE 7th Annual. IEEE, 2016, pp. 1-6.

[31] HealthLine, http://www.healthline.com/health/pregnancy/ what-is-an-obstetrician, [Online; accessed 22-April-2017].

[32] wiseGEEK, http://www.wisegeek.org/what-is-an-obstetrician.htm, [Online; accessed 22-April-2017].

[33] U. of Rochester Medical Center, https://www.urmc.rochester.edu/ encyclopedia/content.aspx?ContentTypeID $=90 \&$ ContentID $=\mathrm{P} 02336$, [Online; accessed 22-April-2017].

[34] S. Pereira, F. Portela, M. F. Santos, J. Machado, and A. Abelha, "Predicting type of delivery by identification of obstetric risk factors through data mining," Procedia Computer Science, vol. 64, pp. 601609, 2015.

[35] K. P. Kornelson, M. Vajjiravel, R. Prasad, P. D. Clark, and T. Najm, "Method and system for developing extract transform load systems for data warehouses," Nov. 21 2006, uS Patent 7,139,779.

[36] "Estatísticas da saúde 2015," Statistics Portugal, Tech. Rep., 2017.

[37] "Hospital maternity activity 2015-16," National Health System, Tech. Rep., 2016.

[38] "Australia's mothers and babies 2014," Australian Institute of Health and Welfare, Tech. Rep., 2016. 